\title{
http://doi.org/10.15359/ree.2002-3.9
}

\section{POBREZA Y EDUCACIÓN}

\author{
M.C José Solano Alpizar* \\ M.Sc. Sandra Ovares Barquero** \\ Licda. Yadira Cerdas Rivera***
}

Este artículo se refiere a cómo la pobreza y la desigualdad crecientes tienen claras expresiones en los resultados educativos, contribuyendo a generar una creciente exclusión social que atenta contra los derechos básicos de los ciudadanos de las zonas rurales del pais.

Las politicas educativas carecen de alternativas para estas zonas y además la inexistencia de un departamento que se encargue de manera específica de la educación rural. Asimismo, los planes de estudio de los profesionales han de estar orientados al desempeño de esta realidad.
This article refers to how increasing poverty and inequality are clearly expressed in the results of education, thus contributing to a growing social exclusion which goes against the basic rights of the citizens in the rural parts of Costa Rica.

The educational policies lack alternatives for these areas; nor is there any department specifically in charge of rural education. In addition, study plans for professionals should be oriented toward achievement within this reality.

* Historiador y pedagogo especialista en Investigaciones Educativas. Subdirector de la División de Educación Rural, autor de Educación y desarrollo en América Latina. jsolano@una.ac.cr

** Máster en Administración Educativa. Ha desarrollado experiencia en curriculum, biología y administración educativa.sovares@yahoo.com

*** Licenciada en Ciencias de la educación. Tiene experiencia en educación primaria. ycerdas@una.ac.cr Los tres autores son profesores e investigadores de la División de Educación Rural y participan del Proyecto "Fortalecimiento de la Educación Rural de Costa Rica y América Central" del CIDE, Universidad Nacional. 


\section{Introducción}

Los actuales cambios en el mundo rural costarricense, acicateados por las políticas de la globalización y reforzados por el impacto de los ajustes estructurales han contribuido considerablemente al incremento de la pobreza en las zonas rurales de nuestro país.

Este incremento se refleja claramente en las estadísticas, mientras que en 1980, la pobreza rural se distribuía por partes iguales entre la región central y la región periférica, en 1989 casi una década después, el $61 \%$ correspondía a la región periférica y el 39\% restante a la región central (Herrero, 1992).

En la decada siguiente, el problema ha alcanzado nuevas dimensiones, como bien lo apunta Sojo (1997), en 1987 el 77\% del total de familias en condición de pobreza que había en el país se ubicaba en zonas rurales, para 1995 este porcentaje se había incrementado notoriamente a un $81 \%$.

Según el Estado de la Nación (2000) nos dice "por zonas tanto en lo urbano como en lo rural, la estimación de 1999 arroja niveles superiores en la pobreza total y, espccialmente, en la pobreza extrema o indigencia. La incidencia de la pobreza sigue siendo mayor en la zona rural.

Estas cifras dejan entrever que se mantiene el patrón histórico de desarrollo. con desventaja para las zonas rurales, a la vez, alertan acerca de la necesidad de forjar un "nuevo paradigma para el desarrollo rural" que no esté atado al desarrollo agrícola sino que contemple, de manera integral, todo un conjunto de ámbitos dentro de los que la educación y lo educativo juegan un papel fundamental.

En este sentido tenemos que mencionar que uno de los factores que ha venido contribuyendo al incremento de los índices de pobreza en América Latina, -y del que Costa Rica no está al margen- es el de la calidad educativa, particularmente la calidad de la educación que es impartida en las zonas rurales.

El deterioro que ha experimentado la educación en las dos últimas décadas se expresa de múltiples maneras, unas más evidentes y notorias que otras, pero igual inciden en el desempeño escolar de las niñas y los niños del medio rural costarricense y contribuyen a la reproducción del círculo vicioso de la pobreza.

Dicho círculo se transmite de generación en generación, tal y como lo senala Rivero (1999), y se traduce en altas tasas de fecundidad en las familias más pobres, mayor riesgo de embarazos mal llevados por las madres de esas familias, en el peor estado nutricional de sus hijos, en las mayores dificultades de éstos para el aprendizaje escolar y, como consecuencia lógica, en mayores tasas de deserción y repetición que obligan a que los desertores y las desertoras terminen trabajando en actividades con poco futuro y de escasa productividad en sectores informales de la economía. 


\section{El círculo vicioso de la pobreza en el contexto rural}

Sí bien es cierto el fenomeno de la globalización ha transformado la sociedad costarricense como un todo, es importante señalar que dada la heterogeneidad estructural, - consecuencia directa del modelo de desarrollo adoptado por la región a partir de la década del cincuenta-, el medio rural ha debido soportar el peso de profundos cambios en distintos ordenes de la vida cultural, social, económica y política.

Se puede mencionar el cambio en los patrones culturales de vida de las familias en el medio rural, tal y como lo señala Román (1997), lo que ha significado cambios en la organización de los sistemas productivos, en la forma de explotación de los recursos naturales, en las estrategias de producción y la participación en las diferentes fases del proceso productivo, industria, comercio, etc. También ha significado cambios en el ámbito social, en términos de las relaciones sociales con otros agentes productivos y con el Estado, y en materia cultural, podemos decir que los nuevos procesos han creado condiciones para que se dé una pérdida de identidad cultural y se desarrollen nuevos valores, actitudes y aptitudes frente a lo tecnológico, el mercado, la información, las inversiones.

De igual manera señala esta autora que ha habido cambios en los hábitos de vida, sean éstos de consumo, períodos de trabajo, plazos de entrega de los productos, períodos de descanso, prácticas de cultivo, y en valores como competencia/solidaridad, riesgo, seguridad en las aspiraciones y las expectativas de desarrollo.

Los programas de ajuste estructural llevados a cabo en la década del ochenta han producido significativos cambios en el panorama rural; es por ello que el medio rural de una situación principalmente agropecuaria se está viendo sometido en este momento a crecientes niveles de competitividad y sostenibilidad como requisito para el desarrollo de su dinámica productiva y sus formas de vida.

En cuestión de dos décadas ha disminuido el empleo agrícola y ha aumentado la inserción laboral en actividades como el comercio y los servicios. Como bien lo apunta el Informe Estado de la Nación (2000) “... el empleo agrícola en la zona rural se redujo de un $48.6 \%$ a un $34,3 \%$ en tanto que el comercio y los servicios aumentaron su participación del $33,4 \%$ al $42,1 \%$ en ese mismo período".

Esta transformación mayormente perceptible en el paso del predominio de actividades agrícolas a un predominio mercantil evidencia que: "el campo es cada vez menos agrícola y se fortalece como un espacio de prestación de servicios tales como el turismo o la microempresa rural y de actividades en expansión" (Informe Estado de la Nación, 2000). 
La disminución del agricultor por cuenta propia - quien ha debido pasar a desempeñar distintas ocupaciones como mano de obra asalariada; sobre todo en actividades no tradicionales de exportación- ha propiciado que éstos pasen a engrosar la lista de los "nuevos pobres", —aunque este concepto aplica para referirse a miembros de clases medias dedicados a la pequeña industria o el comercio de menor rango, creemos que bien podría incluir propietarios agrícolas que han tenido que vender sus pertenencias (fincas, casas, herramientas, etc.) y han debido ofrecerse como mano de obra asalariada en empresas transnacionales y en actividades relacionadas con el área de servicios y de construcción. Son grupos de familias que no eran pobres hasta hace pocos años, y que lo logrado generaciones atrás de manera laboriosa, lo han perdido hasta depender exclusivamente de la venta de su fuerza de trabajo.

En este mismo contexto, el impacto mayor ha recaído sobre la célula básica de la sociedad rural: la familia. Es así como podemos observar la erosión que ha venido expcrimentando la familia en el ámbito rural. Es notorio el aumento de familias incompletas con madres pobres, solas, jefas de hogar; aumento de nacimientos de niños sin reconocimiento del padre y de madres adolescentes que difícilmente van a poder conformar familias orgánicas (Kliksberg: 2000).

Autores como Céspedes y Jiménez (1995) señalan que la incidencia de la pobreza en hogares jefeados por mujeres es mayor que en hogares de jefe masculino, además de que la intensidad de la pobreza es más significativa en el caso de familias que se encuentran bajo la tutela de la mujer.

En este sentido podría argumentarse que a la familia de escasos ingresos cada vez se le presentan mayores dificultades para proporcionar una infancia normal a sus hijos, sobre todo aquellas familias jefeadas por una mujer, éstas han sido mayormente afectadas por el impacto de la pobreza; de ahí que puede hablarse de una feminización de la pobreza.

Esta situación tiene una repercusión determinante en el desarrollo y en las posibilidades de mejoramiento de la calidad de vida de la unidad familiar, al constituir la mujer la figura central en el proceso de socialización de los infantes. Tal y como lo deja asentado el estudio realizado por la CEPAL en 1990 sobre rendimiento escolar en Uruguay “... la madre es el agente socializador fundamental. Desde el comienzo de la vida se comunica con el bebé, comienza a transmitir - vía lenguaje- su nivel cultural y con los actos cotidianos ilustra sobre normas y valores que serán los referentes de la conducta del nin̄o".

En nuestro país, al ser muchas madres jefas de hogar y tener que constituirse en el principal sustento económico de la familia, el patrón de socialización ha venido quedando en manos de los hijos y las hijas mayores, lo que repercute de una u otra manera en el desempeño escolar de unos y otras. 
En este sentido podria argumentarse que a la familia de escasos ingresos cada vez se le presentan mayores dificultades para proporcionar una infancia normal a sus hijos, sobre todo aquellas familias jefeadas por una mujer, éstas han sido mayormente afectadas por el impacto de la pobreza: de ahi que puede hablarse de una feminización de la pobreza.

A lo mencionado se debe sumar el hecho de que ha habido un aumento significativo en el número de niños y niñas menores de catorce años que se han incorporado al campo laboral, lo que los convierte en candidatos naturales para la deserción y la repetición; problemas medulares que enfrenta la educación en el medio rural.

En este sentido, la condición de pobreza creciente que enfientan las familias de las zonas rurales se ve agravada, en la medi$\mathrm{da}$, en que la educación en las zonas rurales no ha venido a constituirse en un verdadero vehículo de movilidad y ascenso social que permita a cada uno de los miembros de la unidad familiar una inserción laboral conducente a un mejoramiento de la calidad de vida, tanto de manera individual como colectiva.

\section{Expresiones educativas de la pobreza en el contexto rural}

La pobreza y la desigualdad crecientes tienen claras expresiones en los resultados educativos, lo que contribuye a generar una creciente exclusión social que atenta contra los derechos básicos de los ciudadanos de las zonas rurales del país.

Este hecho lo podemos observar de manera más clara en la medida en que relacionamos los procesos de desplazamiento de la mano de obra agrícola -a otras actividades- y la forma en que ello afecta el acceso a la educación y el desempeño académico de jóvenes, niñas y niños en este contexto.

La disminución de los agricultores por cuenta propia y el consecuente empobrecimiento de la familia rural como un todo han propiciado la incorporación temprana de los miembros de la familia al mercado laboral, situación que contribuye significativamente a reproducir el circulo vicioso de la pobreza.

Esta inserción temprana en la fuerza de trabajo provoca la deserción de niños y niñas y afecta el rendimiento académico, aspecto que limita el mejoramiento de las condiciones de vida familiar y del contexto ocupacional.

Las estadísticas confirman que la deserción escolar en el medio rural costarricense se ha convertido en uno de los problemas más persistentes. Al respecto 
un estudio sobre la deserción intra-anual realizado por el Departamento de Estadística del Ministerio de Educación Pública (1999) deja entrever datos esclarecedores sobre esta situación, dado que las diferencias numéricas entre la zona urbana y la zona rural son muy marcadas, pues mientras que en la primera se presenta una deserción absoluta de 7554 estudiantes, en la segunda la cifra se duplica a 15.313 cstudiantes.

A lo anterior se debe sumar el incremento de los índices de repitencia que contribuye igualmente a ahondar el problema, pues como lo indican las estadisticas, mientras que la repitencia en primer grado en la zona urbana es de 4694 estudiantes, en la zona rural la cifra asciende a 13683 (Informe Estado de la Nación, 1997).

Este fenómeno de la repitencia es el producto de las cambiantes condiciones de la economía rural, que genera procesos de migración intrarregionales que no se corresponden con las lógicas del sistema educativo, en términos de horarios, métodos, matrículas, evaluación, entre otros aspectos.

La pobreza en las zonas rurales ha estado acompañada, además, por el incremento del analfabetismo funcional -grupos de adultos con escolaridad básica incompleta-, lo que presenta un cuatro más desolador, dado que el analfabetismo constituye una de las máximas expresiones de vulnerabilidad educativa, debido a que el alfabetismo representa uno de los principales indicadores del estado de desarrollo humano de una nación. Se debe tener presente que Rivero (1999) afirma que en Costa Rica el analfabetismo funcional anda alrededor de $40 \%$, a pesar de ser un pais con una alta tasa de alfabetización.

El sistema educativo ha venido contribuyendo a limitar las posibilidades de desarrollo de los jóvenes, las niñas y los niños en las zonas rurales de nuestro pais. Ha coadyuvado - de manera directa o indirecta- al ensanchamiento de la brecha de la desigualdad social, debido a que un individuo que abandona los estudios antes de concluir la primaria, difícilmente podrá acceder a mejores condiciones laborales $y$, por ende, se va a encontrar siempre en desventaja respecto a aquel que sí ha logrado mantenerse en el sistema educativo por más tiempo.

De esta manera, la incidencia de la educación en la reproducción del círculo vicioso de la pobreza se expresa, entre otros aspectos, por medio de:

a) La carencia de políticas educativas específicas para zonas rurales.

b) Un currículo pertinente.

c) La formación de los docentes.

d) La infraestructura educativa.

e) La inflexibilidad del calendario escolar. 


\section{a. La carencia de políticas educativas específicas para las zonas rurales}

Uno de los principales aspectos que contribuye a la exclusión social, vía sistema educativo, es la carencia de políticas educativas específicas para las zonas rurales. Las políticas existentes tienen un carácter uniforme para todo el pais, irrespetando y negando con ello, la particularidad cultural y social de los grupos humanos asentados en el medio rural.

La inexistencia de un departamento que se encargue de manera específica de la educación rural impide que se puedan dar respuestas educativas concretas en términos de presupuesto, atención preescolar, combate de la deserción y la repitencia, salud y nutrición, entre otros.

\section{b. El currículo}

El currículo presenta un sin número de inconvenientes, desde el momento mismo de su elaboración es realizado por personas ajenas a la realidad rural, lo que equivale a que esté desvinculado de las necesidades e intereses de los miembros de estas comunidades.

Esta situación inicial genera falta de pertinencia de los elementos del currículo que se refleja en la planificación educativa (objetivos, contenidos. procedimientos, valores y evaluación) y produce que los procesos educativos que promueve la escuela rural no sean significativos $y$, por ende, útiles para los educandos ni para la comunidad donde viven.

En términos del aprendizaje, este se torna descontextualizado en la medida en que se vuelve más academicista y desvaloriza el conocimiento cotidiano y previo que el niño y la niña poseen desde antes de ingresar a la institución escolar.

\section{c. Los docentes y las docentes}

Uno de los sujetos fundamentales en el proceso educativo es la figura del docente, pues a él o a ella, se le asigna la tarea de orientar los procesos de ensenanza y de aprendizaje.

Actualmente, la figura del docente se encuentra cuestionada, debido a que tanto la sociedad civil como el Estado lo convierten en el único responsable de la "crisis educativa", cuando en realidad es una más de las variables que intervienen en esta problemática.

En el caso de la educación en el medio rural, el docente es -en alguna medida- más una víctima que un responsable de la deficiente calidad educativa. Ello por cuanto la carencia de políticas educativas claras, la ausencia de un 
currículo pertinente, la falta de materiales didácticos, las malas condiciones laborales, la falta de incentivos para estimular su identificación con el medio, entre otros aspectos, se constituyen en factores condicionantes de su quehacer y se reflejan en los resultados educativos de los niños y las niñas que asisten a la institución escolar en dondelos docentes laboran.

Por otro lado, debemos mencionar que uno de los aspectos que influye también en los resultados educativos de sus alumnos tiene relación con la poca identidad del docente con la realidad rural, que en algunos casos se ve reflejada en los prejuicios y el menosprecio que manifiesta durante su práctica pedagógica.

Este aspecto se ve reforzado por el hecho de que a excepción de la Universidad Nacional, la mayor parte de los centros de formación de docentes carecen de planes de formación y de capacitación que contemplen la especificidad de lo rural.

\section{d. La infraestructura educativa}

En términos generales, las escuelas en el medio rural son más pequeñas que las urbanas y seencuentran en situación precaria, debido a la carencia de: mobiliario, recursos materiales, espacio, servicios básicos (electricidad, letrinas, comedor escolar), bibliotecas y recursos económicos, limitaciones que las pone en gran desventaja para que se pueda ofrecer una oferta educativa de calidad.

Estas limitaciones propician el surgimiento de desigualdades, en términos regionales, pues mientras que muchos estudiantes, en otros lugares del país gozan de condiciones básicas para su educación, otros apenas pueden sobrellevar la tarea educativa, debido a la carencia de condiciones mínimas para el logro educativo, lo que se refleja en los resultados de las pruebas nacionales y en los indices de escolaridad.

La falta de compromiso gubernamental para ofrecer los recursos necesarios y la ausencia de una visión prospectiva sobre el papel de la educación en estas zonas, por parte de los políticos, contribuyen significativamente en este creciente proceso de exclusión social.

\section{e. La inflexibilidad del calendario escolar}

Uno de los principales problemas que ha enfrentado la población en el medio rural en el nivel educativo tiene que ver con la inflexibilidad del calendario escolar que, a su vez, se relaciona con la rigidez mental de quienes orientan la política educativa nacional.

A pesar de los resultados positivos que ofrece la propuesta educativa desarrollada en Colombia y que se conoce con el nombre de "escuela nueva" donde 
se plantea una flexibilización del calendario escolar de acuerdo con las actividades económicas de la región, en nuestro país las autoridades aún no comprenden que la realidad económico-social no correspondc con la lógica organizacional del sistema educativo, lo que obliga a una gran cantidad de niños y niñas a abandonar sus estudios en tiempos de cosecha y recolección de fruta, sin que se les presente alternativas educativas al respecto.

El creciente trabajo y contratación de mano de obra temporaria en las zonas rurales exigen la incorporación de toda la familia, que en condiciones de pobreza, se ve obligada a viajar por las distintas regiones del país buscando su sustento.

Todos estos aspectos, unidos a muchos otros que por cucstiones de cspacio no vamos a abordar en este artículo, nutren la creciente exclusión social que se viene experimentando en nuestro país y que se convierten en expresiones educativas de la pobreza. Tal y como lo plantean Bustelo y Minujin (1997), el concepto de exclusión social no es un concepto absoluto sino relativo en un doblc sentido. Por una parte, constituyę la contrapartida de la inclusión, es decir, se está excluido de algo (trabajo, familia, educación, vivienda, afecto, pertencncia comunitaria, entre otros). No se trata de un concepto dicotómico que divide a los individuos en dos; existe una serie de situaciones intermedias cntre ambos estados. Por otro lado, constituye un concepto relativo, en tanto varia en cl ticmpo y el espacio. Por ejemplo, ser analfabeto en nuestros días, constituye un significativo elemento de exclusión social en la "vida moderna", pero no era considerado así en el pasado.

\section{A modo de cierre}

Una de las maneras como se puede romper el circulo vicioso de la pobreza es a través de estrategias sociales y educativas con capacidad de dar respuestas integralcs a las necesidades e intereses de los habitantes de las zonas rurales de nuestro país; pero ello sólo será posible en la medida en que cstas cstrategias se inscriban dentro de una concepción renovable de lo rural.

Esta concepción requiere partir de una revaloración de lo rural que como bien apunta Román (1997) debe contemplar a las familias, las comunidades, sus relaciones y su concepción del mundo, sus valores y sus tradiciones, sus juicios y sus prejuicios, entre otros determinantes, pues esta es una forma de construir un modelo de desarrollo con y para los sujetos involucrados en este proceso social, cultural y económico.

También debe partir de una valoración especial de la educación como eje transversal en cualquier esfuerzo por implementar un modelo de desarrollo 
rural. Hasta ahora, se ha visto lo educativo como un aspecto marginal en las teorizaciones sobre el desarrollo rural, debido a concepciones erróneas sobre el papel y los alcances que la educación puede tener en la formulación de una estrategia.

Quizás, el problema mayor resida en que se piensa lo educativo referido exclusivamente al aula y en lo particular a lo formal institucionalizado, lo que evidencia cierta miopía en relación con la forma en que se debe conceptuar la educación en los ámbitos formal, no formal e informal.

No obstante, que en la década del noventa, en la mayor parte de los documentos y propuestas surgidos en Foros, Cumbres y Conferencias Internacionales, la temática de lo educativo fue una constante a la hora de hablar del desarrollo, sigue estando ausente lo educativo como eje articulador de los procesos conducentes al desarrollo rural. Puede observarse Transformación productiva con equidad: la tarea prioritaria de América Latina y el Caribe (CEPAL, 1990); Conferencia Mundial sobre Educación para Todos, Jomtien, Tailandia (UNESCO, 1990): Educación y conocimiento: eje de la transformación productiva con equidad (CEPAL/UNESCO, 1992); Cumbre Social de Copenhague (1995); V Conferencia Iberoamericana de Educación (OEI, 1995); Priorities and strategies for education (Banco Mundial, 1995); La Educación Encierra un Tesoro (UNESCO, 1996).

Si observamos los fundamentos sobre los que se debe asentar la "nueva ruralidad", éstos parecieran ser comprendidos ignorando el vínculo con lo educativo; de ahí que se hable de desarrollo humano, fortalecimiento de la democracia y la ciudadanía, sostenibilidad, desarrollo del capital social, diversidad cultural, participación, entre otros, como si éstos no se debieran a procesos educativos de variada naturaleza.

La educación en cualquiera de sus manifestaciones constituye uno de los principales pivotes del desarrollo. Para poder trabajar con las comunidades y abordar temas como los de fecundidad, capacitación productiva, género. sostenibilidad, liderazgo local, salud e higiene y organización comunal, entre otros, se requiere de un acercamiento pedagógico que pasa por un reconocimiento del otro tal y como ha sido planteado en las últimas décadas en las propuestas de educación popular.

Es un reconocimiento de la cultura de nuestros interlocutores para coadyuvar al desarrollo de procesos tendientes al mejoramiento de la calidad de vida de las comunidades, no dentro de la lógica clásica del técnico que impone, niega o invisibiliza la cultura, los valores, las necesidades y los intereses de los pobladores del medio rural, sino del que viene con una actitud de respeto y tolerancia de cada microcultura regional. 
En este contexto, la educación debe jugar un papel determinante. Hoy más que nunca tiene que adquirir el carácter de una misión social, partiendo del hecho de que ésta contribuye al fortalecimiento de la identidad cultural, la democracia y la justicia, así como a la autoconfianza individual y a la conservación del medio ambiente (Espinoza, Ooijens, Tampe, 2000).

El desarrollo económico y la equidad sólo podrán lograrse en la medida en que se destinen cada vez más recursos a la educación. Un nuevo "paradigma para el desarrollo rural" debe tener presente en cada uno de sus proyectos el componente educativo, sea en los niveles informal, no formal y formal, pues la educación tiene que convertirse en el eje vertebral de cualquier política de desarrollo rural.

Para que las zonas rurales de nuestro país puedan aspirar a un crecimiento económico en un ambiente de igualdad se requiere dar un salto cualitativo, en términos de la calidad del servicio educativo que se ofrece, mejorando la preparación de los recursos humanos para que puedan insertarse en la estructura productiva con ciertas ventajas competitivas basadas en nuevos y mejores conocimientos, pero sobre todo of reciendo alternativas de formación permanente.

Esta calidad de la que hablamos no está asociada a la promoción de una competencia salvaje que "facilite" un mejor rendimiento escolar. La calidad educativa de la que se habla es aquella que contempla la jerarquización de valores como la cooperación y la solidaridad. Una calidad que se garantiza con condiciones institucionales y laborales aptas para realizar los procesos de enseñanza y de aprendizaje con un curriculo que contemple la diversidad cultural.

Estos son algunos de los retos a los que nos enfrentamos quienes buscamos articular una propuesta de desarrollo rural, en la que se requiere de una visión integral donde laparticipación de los miembros de la comunidad sea real y efectiva, y en la que la educación ocupe el lugar que realmente le corresponde.

\section{Referencias}

Aguilar, M. y Monge, M. (2000). Hacia una pedagogía rural. División de Educación Rural. CIDE, Universidad Nacional. Primera reimpresión. San Josć, Costa Rica: Imprenta Segura Hnos.

Aguilar, M. y otros (2000). Educación rural: un acercamiento pedagsgico. Centro de Investigación y Docencia en Educación. División de Educación Rural. Universidad de Utrecht. Proyecto Fortalecimiento de las Escuelas Rurales de Costa Rica y América Central. San José, Costa Rica: Imprenta Segura Hnos. 
Berry, A. (1999). "Las causas de la pobreza rural en América Latina y políticas para reducirla con referencia especial al Paraguay”. Revista Parciguciyc de Sociologia. Centro paraguayo de estudios sociológicos. Año 36, $N^{\circ} 106$, set.l dic., Asunción.

Céspedes. V. y Jiménez. R. (1995). La pobreza en Costa Rica. San José, Costa Rica.

Delors, J. (1996). La educación encierra un tesoro. Informe a la UNESCO de la Comisión Internacional sobre Educación para el s. XXI. México: UNESCO.

Espinoza, M. Ooijens, J. y Tampe, A. (2000). Educación para el trabajo en áreas rurales de bajos ingresos: una estrategia viable de educación no formal. OIT, CINTERFOR, Montevideo, Uruguay: CINTERFOR.

Grynspan, R. (1997). La pobreza en América Latina y estrategias para superarla. Debate Contemporáneo.

Herrero, F. (1992). "Ajuste y pobreza rural en la década de los ochenta: el caso de Costa Rica". Trejos, R. (editor) Ajuste macroeconómico en América Laiina. Programa l. Análisis y planificación de la política agraria. San José, Costa Rica: IICA.

IICA (1999). Nueva ruralidad. El desarrollo rural sostenible en el marco de una nueva lectura de la ruralidad. Dirección de Desarrollo Rural Sostenible. San José, Costa Rica.

Klisksberg, B. (2000). "América Latina: una región en riesgo. Pobreza, inequidad e institucionalidad social”. Revista Economia y Sociedad. Vol. $40 \mathrm{~N}^{\circ}$ 14, set./dic. Heredia: Universidad Nacional.

Korzeniewicz, R. y Smith, W. (2000). "Pobreza, desigualdad y crecimiento en América Latina: en búsqueda del camino superior a la globalización". Desarrollo económico, Vol. $40 \mathrm{~N}^{\circ} 159$, octubre/diciembre.

Ministerio de Educación Pública (1999). Deserción intra-cunucle en el sistema educativo. División de Planeamiento y Desarrollo Educativo. Departamento de Estadística. San José: MEP. 
OEI (1995). "La educación como factor de desarrollo". V Conferencia Iberoamericana de Educación. Buenos Aires.

Pérez, L. y Pichardo, A. (1994). Pobreza en el istmo centroamericano: perspectiva de las mujeres. Tomo II. PNUD. San José: PNUD.

Pomareda, C. (1997). "Lo rural: una visión optimista". Desarrollo rural sostenible en Costa Rica: Avances y perspectivas. San José, Costa Rica: Editorial Porvenir.

Proyecto Estado de la Nación (1997). Informe Estado de la nación en Desarrollo Humano Sostenible. Cuarto Informe. San José, Costa Rica.

Proyecto Estado de la Nación (1999). Estado de la Región en Desarrollo Humano Sosteniblc. Primer Informe. San José, Costa Rica.

Renzi, M. y Kruijt, D. (1997). Los nuevos pobres. San José Costa Rica: FLACSO.

Rivero. J. (1999). "Educación y pobreza: políticas, estrategias y desafios". Revista Paraguaya de Sociologia. Centro paraguayo de cstudios sociológicos. Año 36. N 106, sct./dic., Asunción.

Román, I. (1997) "Tendencias de cambio social en el proceso de modernización agrícola costarricense (1980-1996)". Revista Perspectivas Rurales. Heredia. Universidad Nacional.

Sojo, C. (1997). Los de en medio. Facultad Latinoamericana de Ciencias Sociales, San José, Costa Rica: FLACSO.

Trejos, R. (1992). Ajuste macroeconómico y pobreza rural en América Latina. San José, Costa Rica: IICA.

UNESCO (1994). "Educación para todos: una visión amplia". Conferencia Mundial sobre Educación para Todos. Jomtien, Tailandia: UNESCO. 